\title{
Antioxidant Activities of Plumbagin and Its Cu (II) Complex
}

\author{
Mingxiong Tan, ${ }^{1,2}$ Yancheng Liu, ${ }^{2}$ Xujian Luo, ${ }^{2}$ Zhenfeng Chen, ${ }^{2}$ and Hong Liang ${ }^{2}$ \\ ${ }^{1}$ Department of Chemistry and Biology, Yulin Normal College, Yulin 537000, China \\ ${ }^{2}$ The Key Laboratory for the Chemistry and Molecular Engineering of Medicinal Resources of Ministry of Education, \\ School of Chemistry, Chemical Engineering, Guangxi Normal University, Guilin 541004, China \\ Correspondence should be addressed to Mingxiong Tan, tanmx00@163.com
}

Received 31 July 2011; Accepted 19 August 2011

Academic Editor: Zhe-Sheng Chen

Copyright () 2011 Mingxiong Tan et al. This is an open access article distributed under the Creative Commons Attribution License, which permits unrestricted use, distribution, and reproduction in any medium, provided the original work is properly cited.

Plumbagin and its $\mathrm{Cu}$ (II) complex [Cu (plumbagin $\left.)_{2}\right] \cdot \mathrm{H}_{2} \mathrm{O}$ have been synthesized, and their antioxidant activities towards the inhibitory effect on DPPH free radical, reducing power, total antioxidant capacity, and inhibition on lipid peroxidation were investigated. Plumbagin and its $\mathrm{Cu}$ (II) complex were found to exhibit scavenging activities on DPPH radical with the inhibitory rate of $41 \%$ and $24 \%$, respectively. The reducing power of plumbagin was outstanding at the concentrations of 1.0 , 1.5 , and $2.0 \mathrm{mg} / \mathrm{mL}$, compared to $\mathrm{Cu}$ (II) complex and synthetic antioxidant 2,6-di-ter-butyl-4-methylphenol (BHT); the highest level reached 1.333 for plumbagin and 0.581 for $\mathrm{Cu}$ (II) complex. Also, the inhibition on lipid peroxidation of plumbagin was higher than that of $\mathrm{Cu}$ (II) complex and BHT, $46.4 \%$ for plumbagin and $24.5 \%$ for $\mathrm{Cu}$ (II) complex. The results give a strong impact for designing anticancer drugs, combined with their potential cytotoxic and antioxidant activities, which can be targeted selectively against cancer cells and increase their therapeutic index and additional advantages over other anticancer drugs.

\section{Introduction}

Recently, a large number of metal complexes have been designed and tested for anticancer activity and for supportive therapy in cancer patients, including the anticancer drugs doxorubicin, mitoxantrone, bleomycin, and hydroxyurea [1$3]$. There is a major debate at present that natural products with diverse bioactivities are becoming an important source of potential (pro)drug chelators $[4,5]$. Many naturally occurring molecules such as flavonoids, phenols, and quinones have metal chelating properties, cytotoxic, and antioxidant properties, including the inhibitory effect on free radicals and other damaging oxygen activated products such as the hydroxyl radical, superoxide, hydrogen peroxide, and lipid peroxides $[6,7]$. Thus, the prospect of designing chelating prodrugs can be targeted selectively and activated against cancer cells combined with their potential cytotoxic and antioxidant activities increasing their therapeutic index and provides additional advantages over other anticancer drugs [8-10].

Our earlier studies have reported the synthesis and anticancer activities of plumbagin (Scheme 1) and its $\mathrm{Cu}$ (II) complex (Scheme 2). Plumbagin structurally derived from naphthoquinone was extracted from Plumbago zeylanica $L$, a Chinese traditional medicine. It was found that both plumbagin and its $\mathrm{Cu}$ (II) complex exhibited significant antitumor activities against seven human tumour cell lines (BEL-7404, NCI-H460, CNE-2, 786-O, MCF-7, HCT-116, and Hep$\mathrm{G} 2$ ), and $\mathrm{Cu}$ (II) complex possessed significantly enhanced antitumor with respect to the free ligand $\mathrm{Cu}$ (II) ion plays an important role [11]. All of these results make it interesting to study the antioxidant activities of plumbagin and its $\mathrm{Cu}$ (II) complex.

\section{Materials and Methods}

UV-Vis absorption spectra were performed on a Varian Cary100 UV-Visible spectrophotometer. 2,2' -Diphenyl-1-picrylhydrazyl (DPPH) was purchased from Sigma Chemicals Co. All the metallic salts were purchased from Alfa Co. Ltd. The solvents were analytical grade. All the materials were used as received without further purification unless noted specifically.

Plant material, roots of $P$. zeylanica, was collected in Guangxi province and identified by Prof. Tang (Institute of Life Science, Guangxi Normal University). 


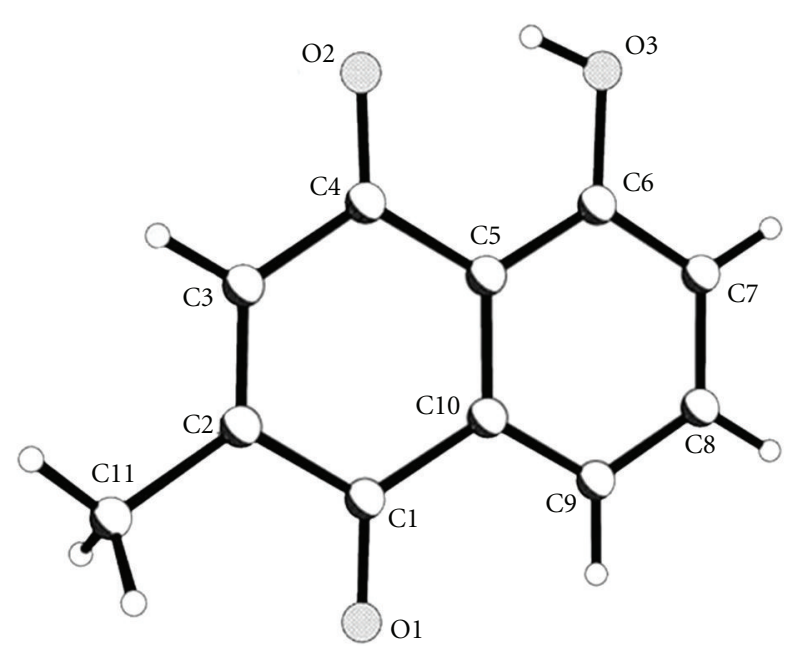

SCHeme 1: Crystal structure of plumbagin.

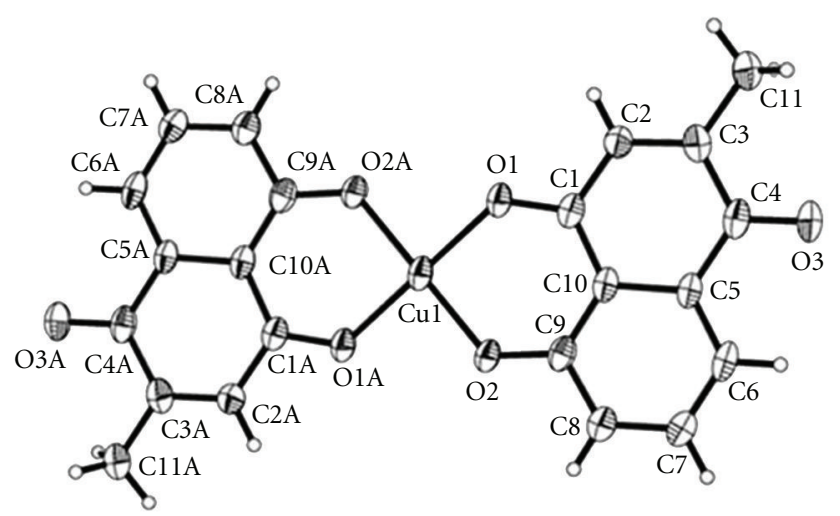

Scheme 2: Crystal structure of plumbagin Cu (II) complex.

2.1. Isolation and Structure Identification of PLN. The roots of $P$. zeylanica $(15 \mathrm{~kg}$ ) were extracted with $95 \% \mathrm{EtOH}$. The solvent was concentrated in vacuo, and the residue was successively partitioned between $\mathrm{H}_{2} \mathrm{O}$ and n-hexane followed by EtOAc. The EtOAc extract ( $252 \mathrm{~g}$ ) was subjected to silica gel cc with a gradient of EtOAc in n-hexane, eluting with nhexane-EtOAc $(9: 1)$ to yield an orange crystal, plumbagin (1.33 g).

2.2. Synthesis of $\left.[\mathrm{Cu} \text { (Plumbagin })_{2}\right] \cdot \mathrm{H}_{2} \mathrm{O}$. This yellowish brown of plumbagin $\mathrm{Cu}$ (II) complex was synthesized by the same method as the reference described by Chen et al. [11].

2.3. Scavenging Activity on DPPH Radical. To evaluate the free radical scavenging activity, plumbagin or $\mathrm{Cu}$ (II) complex solution was allowed to react with a stable free radical, 2,2'-diphenyl-1-picrylhydrazyl radical (DPPH) [12]. $1 \mathrm{mg} / \mathrm{mL}$ of plumbagin or $\mathrm{Cu}$ (II) complex in different reaction times $(10,20,30,40,50$, and $60 \mathrm{~min})$ was assayed, and $1 \mathrm{mg} / \mathrm{mL}$ of synthetic antioxidant 2,6-di-ter-butyl-4methylphenol (BHT) used for comparison. The reaction mixture was incubated at $25^{\circ} \mathrm{C}$. The scavenging activity on
DPPH radical was determined by measuring the absorbance at $515 \mathrm{~nm}$ each $10 \mathrm{~min}$ until the reaction reached the steady state. The antioxidant activity was expressed as a percentage of scavenging activity on DPPH radical: $\mathrm{SC} \%=[1-$ (absorbance of sample)/(absorbance of control) $] \times 100 \%$.

2.4. Reducing Power. The determination of reducing power was performed as described by Oyaizu [13]. The reducing power of various plumbagin and $\mathrm{Cu}$ (II) complex solutions $(0.5,1.0,1.5$, and $2.0 \mathrm{mg} / \mathrm{mL})$ was investigated; BHT was also determined for comparison. The various samples were mixed with phosphate buffer $(2.5 \mathrm{~mL}, 0.2 \mathrm{M}, \mathrm{pH} 6.6)$, potassium ferricyanide $(2.5 \mathrm{~mL}, 1 \%)$ was incubated at $25^{\circ} \mathrm{C}$ for $20 \mathrm{~min}$, trichloroacetic acid was added, and the mixture was centrifuged at $3000 \mathrm{rpm}$ for $10 \mathrm{~min}$. The upper layer of solution $(2.5 \mathrm{~mL})$ was mixed with distilled water $(2.5 \mathrm{~mL})$ and ferric chloride $(0.5 \mathrm{~mL}, 0.1 \%)$, then the absorbance was measured at $695 \mathrm{~nm}$ against a blank.

2.5. Determination of Total Antioxidant Capacity. The total antioxidant capacity was determined according to the method of Prieto [14]. Plumbagin or $\mathrm{Cu}$ (II) complex $(0.5 \mathrm{mg} / \mathrm{mL})$ in different reaction times $(20,40,60,80$, 100 , and $120 \mathrm{~min}$ ) was combined with $0.3 \mathrm{~mL}$ of reagent solution $(0.6 \mathrm{M}$ sulphuric acid, $28 \mathrm{mM}$ sodium phosphate, and $4 \mathrm{mM}$ ammonium molybdate). The reaction mixture was incubated at $95^{\circ} \mathrm{C}$ for $150 \mathrm{~min}$. After the mixture had been cooled to room temperature, the absorbance of the mixture was measured at $595 \mathrm{~nm}$ against a blank. The readings were taken each $30 \mathrm{~min}$. The antioxidant activity of BHT $(0.5 \mathrm{mg} / \mathrm{mL})$ was also assayed for comparison.

2.6. Antioxidant Potential of Plumbagin or Cu (II) Complex in Peanut Oil. Calculated amounts of plumbagin or Cu (II) complex solution were added to $50 \mathrm{~mL}$ of peanut oil. The additive was mixed into the oil with a magnetic stirrer. The oxidative deterioration of samples was studied using Schaal oven test method as described by Economou et al. [15]. The oil samples $(50 \mathrm{~mL}$ each) were placed in open $100 \mathrm{~mL}$ beakers and placed in $60 \pm 0.5^{\circ} \mathrm{C}$ oven for $24 \mathrm{~h}$. The sample $(1 \mathrm{mg} / \mathrm{mL})$ was prepared under the same conditions treated with different reaction times $(1,2,3,4,5$, and 6 days), BHT ( $1 \mathrm{mg} / \mathrm{mL}$ ) was also assayed for comparison. The rate of antioxidant of peanut oil was estimated according to the increase of 2-thiobarbituric acid-reactive substances (TBARS) using the classical TBA procedure. The TBARS values of untreated and treated samples were used to calculate the inhibition of lipid oxidation as follows: Inhibition (\%) = (control-treatment)/control $\times 100 \%$.

2.7. Statistical Analysis. All experimental results were centred at using three parallel measurements of mean \pm SD. Analysis of variance was performed by ANOVA procedure. Duncan's new multiple-range test was used to determine the differences of means. $P$ values $<0.05$ were regarded as significant and $P$ values $<0.01$ as very significant. 


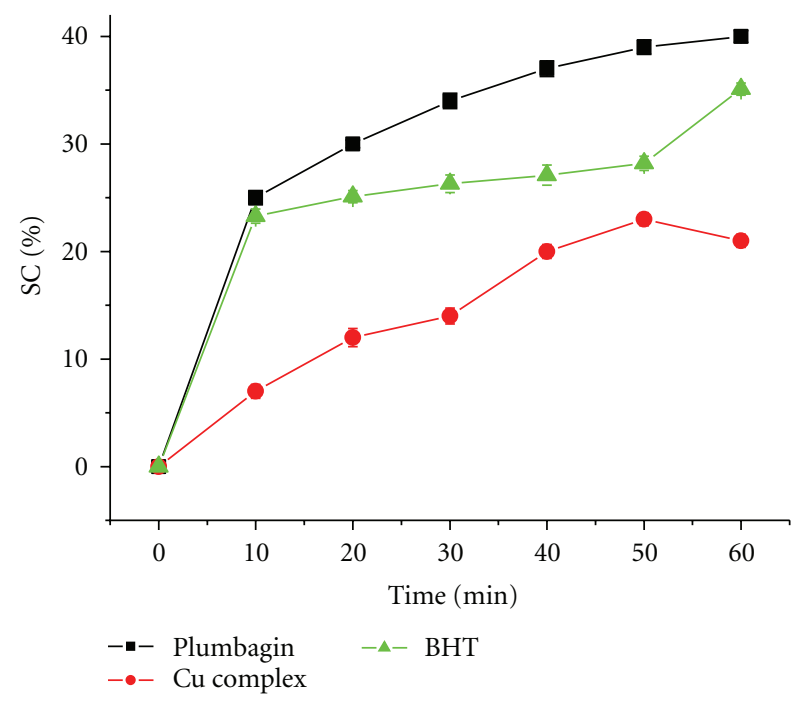

FIGURE 1: DPPH free radical scavenging activity of plumbagin, $\mathrm{Cu}$ (II) complex, and BHT in different reaction times (10, 20, 30, 40, 50 , and $60 \mathrm{~min}$ ).

\section{Results and Discussion}

3.1. Scavenging Activity on DPPH Radical. Antioxidant properties, especially radical scavenging activities, are very important due to the deleterious role of free radicals in biological system. DPPH is a kind of stable free radical and accepts an electron or hydrogen radical to become a stable diamagnetic molecule [6], which was widely used to investigate radical scavenging activity now for its advantage of ease and economy. In DPPH radical scavenging assay, antioxidants are able to reduce the stable DPPH radical to yellow-coloured, and the antioxidant power is indicated by the degree of discoloration which could be determined by measuring of a decrease in the absorbance at $515 \mathrm{~nm}$.

As Figure 1 shows, at the concentration of $1 \mathrm{mg} / \mathrm{mL}$ of plumbagin or $\mathrm{Cu}$ (II) complex possessed scavenging activities on DPPH radical with the inhibitory rate of $41 \%$ and $24 \%$, respectively, their scavenging effect increased with increasing reaction time, and plumbagin scavenging activities are pretty, more higher than its $\mathrm{Cu}$ (II) complex and $\mathrm{BHT}$, indicating that the presence of $\mathrm{Cu}$ (II) decreased the scavenging effect of DPPH radical.

3.2. Reducing Power. The reducing capacity of a compound may serve as a significant indicator of its potential antioxidant activity [16]. In the assay, the presence of reductants in the antioxidant sample causes the reduction of the $\mathrm{Fe}^{3+}$ /ferricyanide complex to the $\mathrm{Fe}^{2+}$ /ferrous form [17], so the reducing power of the sample could be monitored by measuring the formation of Perl's Prussian blue at $695 \mathrm{~nm}$ [18]. As Figure 2 shows, the reducing properties of plumbagin and $\mathrm{Cu}$ (II) complex at different concentration exhibited correlation with increasing concentration. The reducing power of plumbagin was outstanding at the concentration of 1.5 and $2.0 \mathrm{mg} / \mathrm{mL}$ compared to $\mathrm{Cu}$ (II) complex and BHT. At the concentration of $2.0 \mathrm{mg} / \mathrm{mL}$, the reducing power of

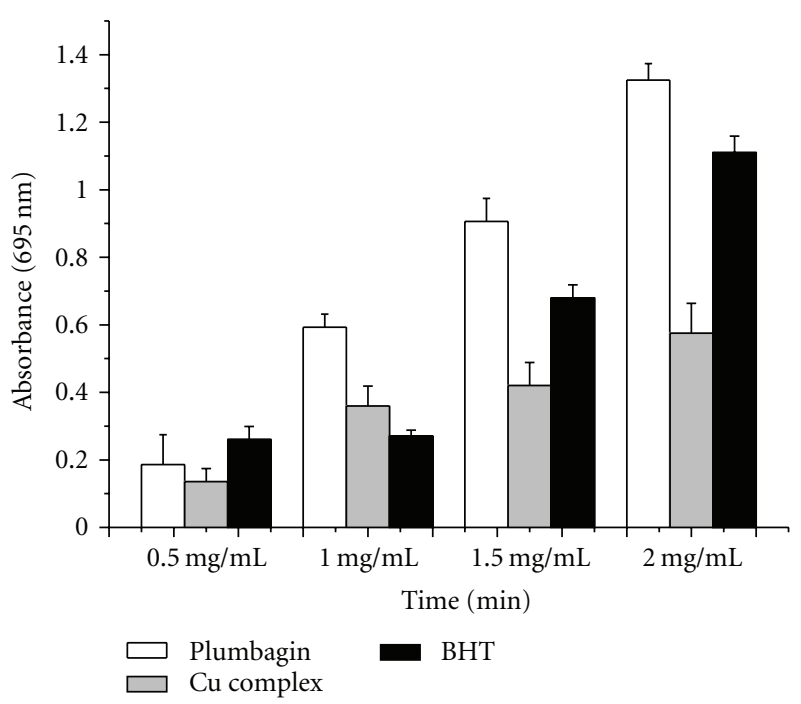

FIgURE 2: The reducing power of plumbagin, $\mathrm{Cu}$ (II) complex and BHT treated with different solution concentrations $(0.5,1.0,1.5$, and $2.0 \mathrm{mg} / \mathrm{mL}$ ).

plumbagin and $\mathrm{Cu}$ (II) complex reached 1.333 and 0.581 , respectively. It was speculated that the decreased antioxidant power of $\mathrm{Cu}$ (II) complex, compared to plumbagin, may be the absence of phenolic group due to forming the complex, thus the presence of the active hydrogen in phenolic hydroxyl group was likely to contribute considerably to the observed antioxidant effects.

3.3. Total Antioxidant Capacity Assay. The assay is based on the reduction of Mo (VI) to Mo (V) by plumbagin and $\mathrm{Cu}$ (II) complex in different reaction times $(20,40,60,80$, 100 , and $120 \mathrm{~min}$ ) and subsequent formation of a green phosphate/Mo (V) complex at acid $\mathrm{pH}$. The high absorbance values indicated that the sample possessed significant antioxidant activity. As Figure 3 shows, plumbagin and $\mathrm{Cu}$ (II) had significant total antioxidant activities compared to BHT, and the effects increased with increasing reaction time, while total antioxidant capacity of $\mathrm{Cu}$ (II) complex was superior to plumbagin.

3.4. Lipid Peroxidation in Peanut Oil. The level of 2-thiobarbituric acid-reactive substances (TBARS), products of lipid peroxidation, is often measured in order to assess the extent of oxidation that occurs in biological systems. Peanut oil was treated with plumbagin, $\mathrm{Cu}$ (II), and BHT complex at the concentration of $1 \mathrm{mg} / \mathrm{mL}$ in different reaction times $(1,2,3,4,5$, and 6 days). As shown in Figure 4, the inhibition rate of lipid oxidation between plumbagin and $\mathrm{Cu}$ (II) complex had significant effects and was enhanced with increasing reaction time, the highest level reached $46.4 \%$ for plumbagin and $24.5 \%$ for Cu (II) complex after 6 days, and the lipid oxidation inhibition of $\mathrm{Cu}$ (II) complex is lower than that of PLN and BHT at the same period. 


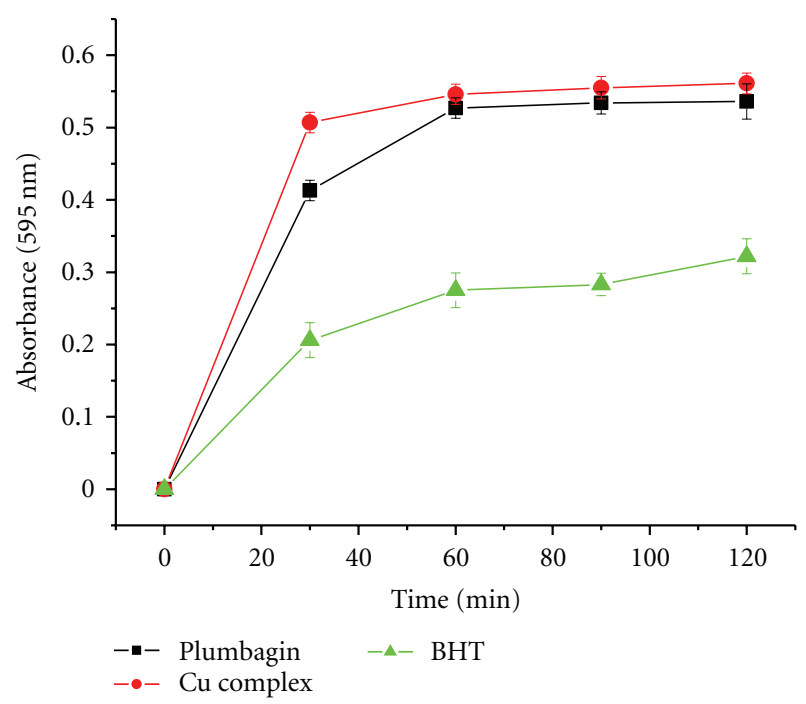

Figure 3: The total antioxidant power of plumbagin, $\mathrm{Cu}$ (II) complex, and BHT in different reaction times $(20,40,60,80,100$, and $120 \mathrm{~min}$ ).

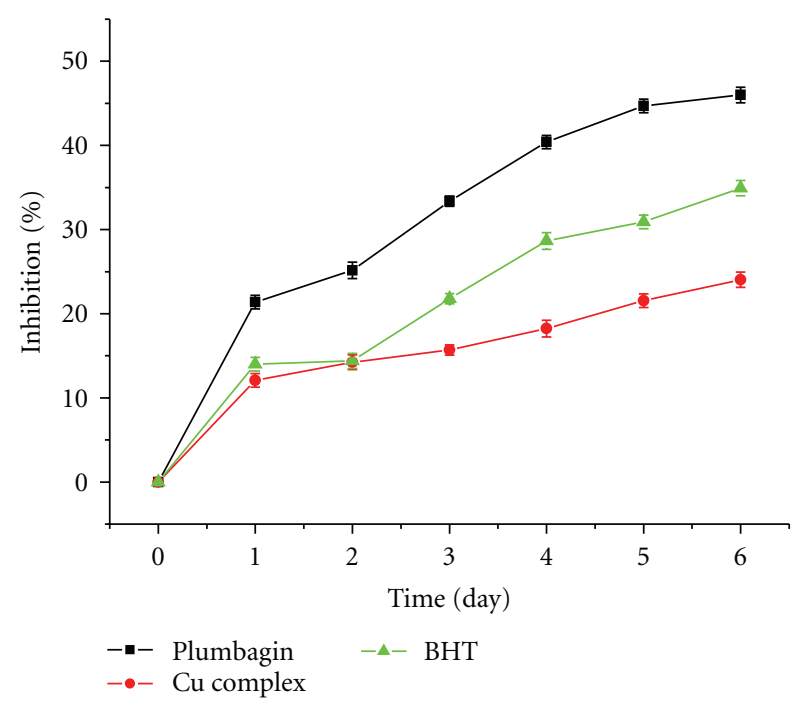

FIgURE 4: Lipid oxidation inhibition by plumbagin, Cu (II) complex, and BHT in peanut oil in different reaction times $(1,2,3,4,5$, and 6 days).

\section{Conclusions}

In this study, we have investigated the antioxidant possibility of plumbagin and its $\mathrm{Cu}$ (II) complex. The results indicated that both the compounds exhibited some antioxidant activities, including the inhibitory effect on DPPH free radical, reducing power, total antioxidant capacity, and lipid peroxidation, which plumbagin possessed higher abilities than that of $\mathrm{Cu}$ (II) complex and BHT at the same condition towards DPPH free radical, reducing power, and lipid peroxidation. It was speculated that the decreased antioxidant power of $\mathrm{Cu}$ (II) complex compared to plumbagin may be the absence of phenolic group due to forming the complex, thus the presence of the active hydrogen in phenolic hydroxyl group was likely to contribute considerably to the observed antioxidant effects. Also, this study gives a strong impact for designing anticancer drugs, combined with their potential cytotoxic and antioxidant activities, which can be targeted selectively against cancer cells and increase their therapeutic index and additional advantages over other anticancer drugs.

\section{Acknowledgments}

This work was supported by the National Natural Science Foundation of China (no. 81060360), the Natural Science Foundation of Guangxi Province (no. 2010-013064 and 2009-0991284), the Key Laboratory for the Chemistry and Molecular Engineering of Medicinal Resources (Guangxi Normal University), Ministry of Education of China (no. CMEMR2011-09), and the Scientific Researches Foundation of Educational Committee of Guangxi Province (no. 200808LX407).

\section{References}

[1] J. Blatt, S. R. Taylor, and G. J. Kontoghiorghes, "Comparison of activity of deferoxamine with that of oral iron chelators against human neuroblastoma cell lines," Cancer Research, vol. 49, no. 11, pp. 2925-2927, 1989.

[2] G. J. Kontoghiorghes, K. Pattichis, K. Neocleous, and A. Kolnagou, "The design and development of deferiprone (L1) and other iron chelators for clinical use: targeting methods and application prospects," Current Medicinal Chemistry, vol. 11, no. 16, pp. 2161-2183, 2004.

[3] M. F. Primik, G. Mühlgassner, M. A. Jakupec et al., "Highly cytotoxic copper(II) complexes with modified paullone ligands," Inorganic Chemistry, vol. 49, no. 1, pp. 302-311, 2010.

[4] L. Wang, S. Gou, Y. Chen, and Y. Liu, "Potential new antitumor agents from an innovative combination of camphorato, a ramification of traditional Chinese medicine, with a platinum moiety," Bioorganic and Medicinal Chemistry Letters, vol. 15, no. 14, pp. 3417-3422, 2005.

[5] L. Kuo-Hsiung, "Discovery and development of natural product-derived chemotherapeutic agents based on a medicinal chemistry approach," Journal of Natural Products, vol. 73, no. 3, pp. 500-516, 2010.

[6] K. Slinkard and V. L. Singleton, "Total phenol analyses: automation and comparison with manual methods," American Journal of Enology and Viticulture, vol. 28, no. 1, pp. 49-55, 1977.

[7] M. X. Tan, J. C. Zhu, Y. M. Pan, and H. S. Wang, "Synthesis, cytotoxic activity, and DNA binding properties of copper (II) complexes with hesperetin, naringenin, and apigenin," Bioinorganic Chemistry and Applications, vol. 2009, Article ID 347872, 2009.

[8] G. K. Kontoghiorghes, M. I. Jackson, and J. Lunec, "In vitro screening of iron chelators using models of free radical damage," Free Radical Research Communications, vol. 2, no. 12, pp. 115-124, 1986.

[9] S. Kummar, M. Gutierrez, and J. H. Doroshow, "Drug development in oncology: classical cytotoxics and molecularly targeted agents," British Journal of Clinical Pharmacology, vol. 62 , no. 1, pp. 15-26, 2006.

[10] S. Blois, "Antioxidant determinations by the use of a stable free radical," Nature, vol. 181, no. 4617, pp. 1199-1200, 1958. 
[11] Z. F. Chen, M. X. Tan, L. M. Liu, H. Liang, and O. Chris, “Cytotoxicity of the traditional chinese medicine (TCM) plumbagin in its copper chemistry," Dalton Transactions, vol. 28, no. 48, pp. 10824-10833, 2009.

[12] C. Sanchez-Moreno, J. A. Larrauri, and F. Saura-Calixto, "A procedure to measure the antiradical efficiency of polyphenols," Journal of the Science of Food and Agriculture, vol. 76, no. 2, pp. 270-276, 1998.

[13] M. Oyaizu, "Studies on product of browning reaction prepared from glucose amine," Japanese Journal of Nutrition, vol. 44, pp. 307-315, 1986.

[14] P. Prieto, M. Pineda, and M. Aguilar, "Spectrophotometric quantitation of antioxidant capacity through the formation of a phosphomolybdenum complex: specific application to the determination of vitamin E," Analytical Biochemistry, vol. 269, no. 2, pp. 337-341, 1999.

[15] K. D. Economou, V. Oreopoulou, and C. D. Thomopoulos, "Antioxidant activity of some plant extracts of the family labiatae," Journal of the American Oil Chemists Society, vol. 68, no. 2, pp. 109-113, 1991.

[16] A. Yildirim, A. Mavi, M. Oktay, A. A. Kara, F. Algur, and V. Bilaloglu, "Comparison of antioxidant and antimicrobial activities of Tilia (Tilia argentea Desf ex DC), sage (Salvia triloba L.), and Black tea (Camellia sinensis) extracts," Journal of Agricultural and Food Chemistry, vol. 48, no. 10, pp. 50305034, 2000.

[17] I. Gülcin, "Antioxidant and antiradical activities of L-carnitine," Life Sciences, vol. 78, no. 8, pp. 803-811, 2006.

[18] Y. C. Chung, C. T. Chang, W. W. Chao, C. F. Lin, and S. T. Chou, "Antioxidative activity and safety of the 50\% ethanolic extract from red bean fermented by Bacillus subtilis IMRNK1," Journal of Agricultural and Food Chemistry, vol. 50, no. 8, pp. 2454-2458, 2002. 


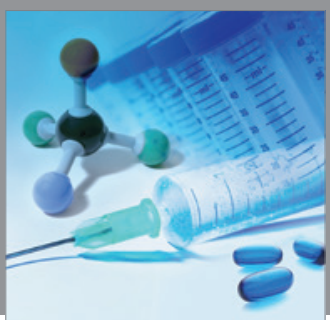

International Journal of

Medicinal Chemistry

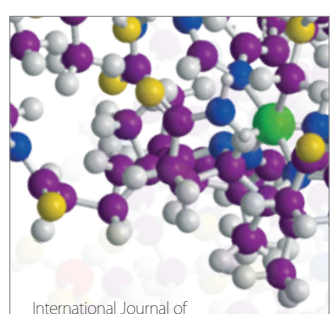

Carbohydrate Chemistry

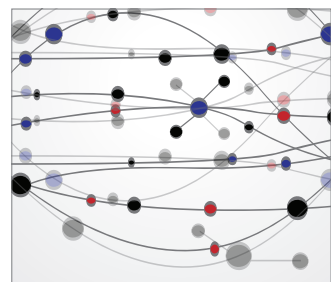

The Scientific World Journal
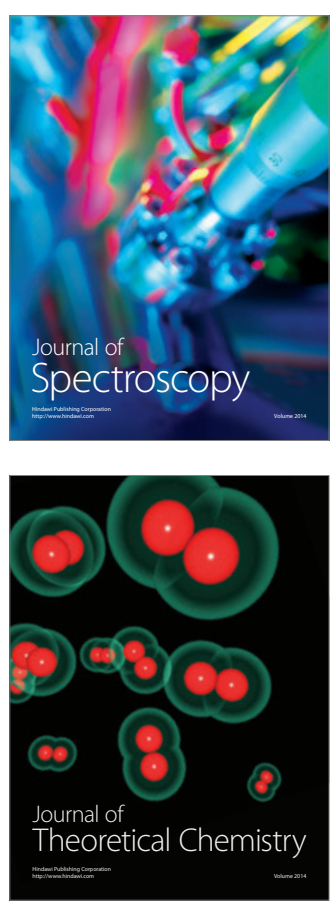
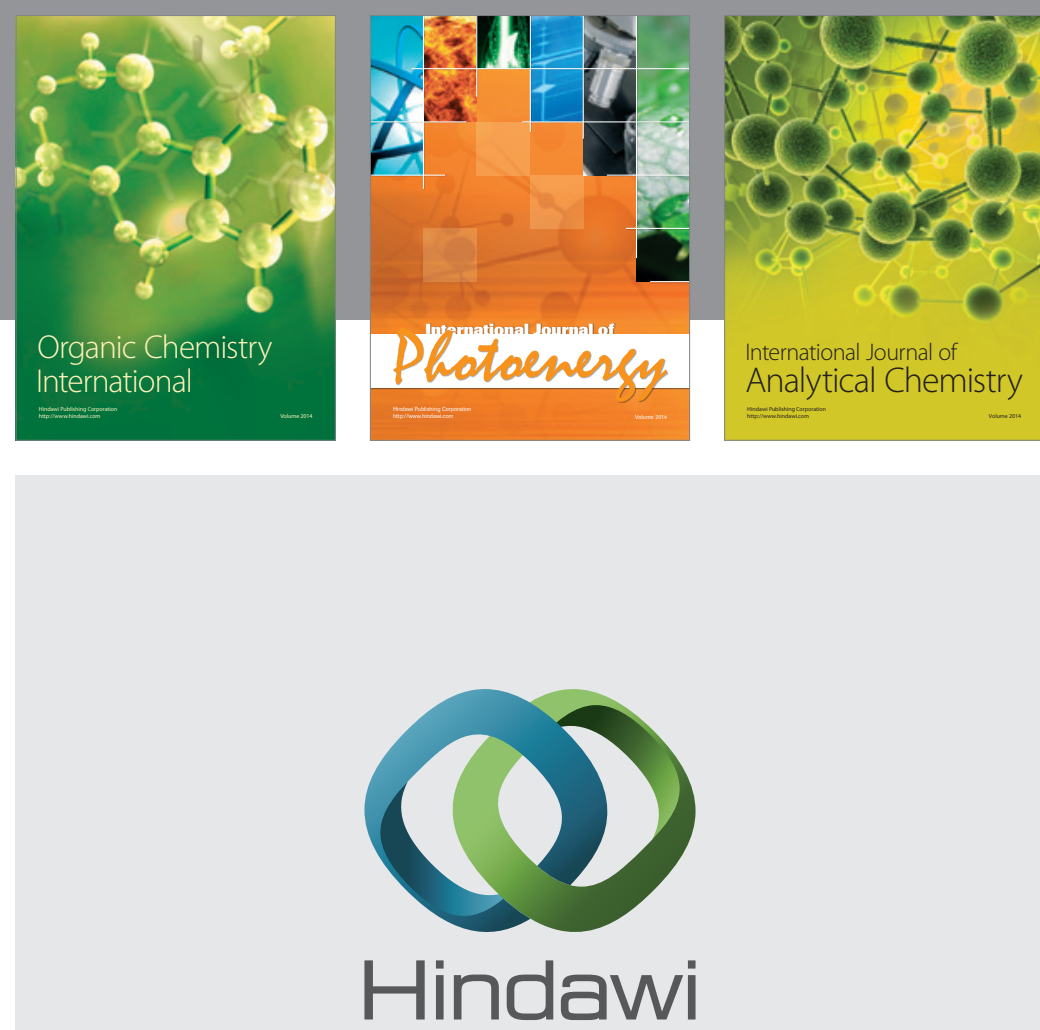

Submit your manuscripts at

http://www.hindawi.com
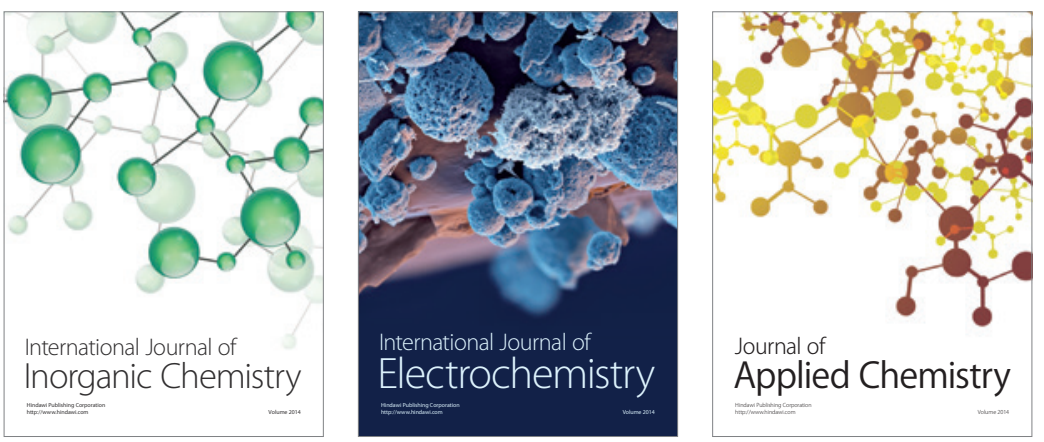

Journal of

Applied Chemistry
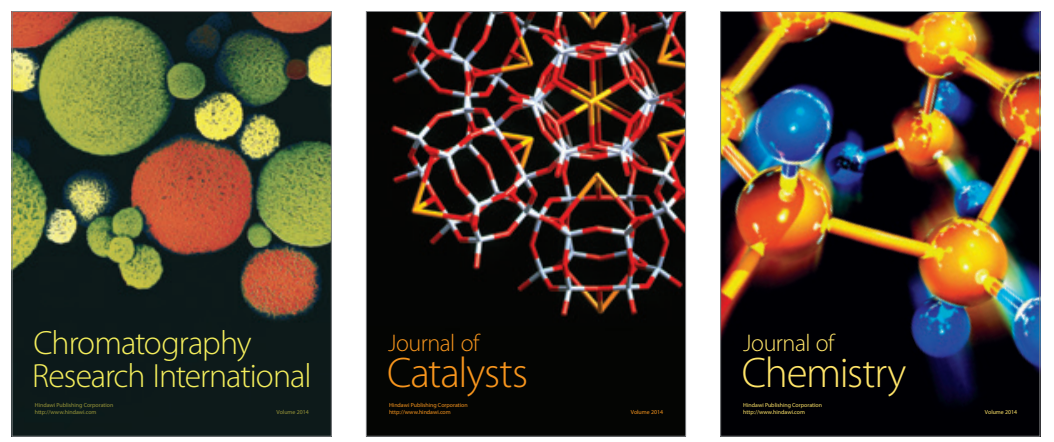
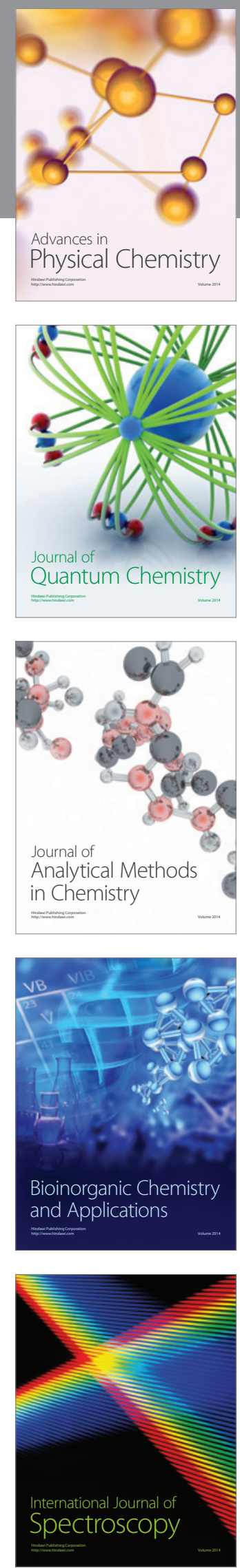